\title{
Verhalten der isoliert perfundierten Rattenleber nach Galaktosaminvergiftung
}

\author{
Von H.-J. MIERTSCh und L. RókA \\ Aus dem Institut fïr Klinische Chemie (Leiter: Prof. Dr. L'. Róka) der Justus Liebig Unìiersität Gießen
}

(Eingegangen am 2. Februar 1972/17. Juli 1972)

\begin{abstract}
Lebern von Ratten, die Galaktosamin erhalten haben, zeigen bei der isolierten Perfusion charakteristische Schädigungen. Insbesondere eine verminderte Gallenproduktion und verminderte Bromthalein-Clearance durch die Galle, eine herabgesetzte Eliminationsfähigkeit von Ammoniumionen aus dem Perfusat, verbunden mit einer eingeschränkten Harnstoffsynthese, Unfähigkeit, einen Glucosespiegel im Perfusat einzuregulieren und vermehrte Abgabe von Kalium sowie cytoplasmatischer und mitochondrialer Enzyme aus den I.eberzellen an das Perfusat.

Führt man dem Perfusat Glucose zu, dann werden bei einer Reihe dieser Leistungen signifikante Verbesserungen erreicht. Die beobachteten Schäden sind offenbar darauf zurückzuführen, daß durch die Blockade der Glykogensynthese keine Glykogenreserven mehr zur
\end{abstract} Verfügung stehen, so daß die Leber für ihre Leistungen auf exogen zugeführte Glucose angewiesen ist.

\section{The bebaviour of the isolated perfused rat liver after poisoning with galactosamine}

Rats were fed galactosamine and the livers taken for isolated perfusion experiments. The perfused livers showed characteristic damage, especially in a decreased production of bile with a decreased clearance of bromosulphophthalein via the bile, a decreased ability to remove ammonium ions from the perfusate, related to a decreased synthesis of urea, a lack of ability to regulate the level of glucose in the perfusate, and the increased loss of potassium and of cytoplasmic and mitochondrial enzymes from the liver cells into the perfusate.

A number of these deficiencies vere significantly counteracted by the addition of glucose to the perfusate.

The observed damage apparently results from the blockage of glycogen synthesis. This causes the absence of any glycogen reserves so that the liver becomes dependent on an exogenous supply of glucose.

Durch die intravenöse oder intraperitoneale Gabe von Galaktosamin lassen sich bei Ratten Veränderungen an der Leber hervorrufen, die der menschlichen Hepatitis ähneln (1). Wir haben geprüft, wieweit man an der isoliert perfundierten Rattenleber, die seit MrLLER (2) und Schimassek (3) als brauchbares Modell für das Studium verschiedener Leberfunktionen benutzt wird, funktionelle Veränderungen nach Galaktosaminvergiftung nachweisen kann.

Wir haben bestimmt:

1. Die Syntheseleistung anhand der Harnstoffsynthese.

2. Die Regulation des Glucosespiegels im Perfusat.

3. Die Gallenproduktion und die Bromthalein-(BSP)Clearance durch die Galle.

4. Die Elimination von Ammoniak aus dem Perfusat und schließlich

5. die Zellschädigung gemessen an der Abgabe von Kalium sowie cytoplastischer und mitochondrialer Enzyme aus den Leberzellen an das Perfusat.

\section{Material und Methodik}

Wir verwendeten normal ernährte Wistar-Ratten im Gewicht von $180-260 \mathrm{~g}$. Zur Galaktosaminvergiftung erhielten die Tiere $24 \mathrm{~h}$ vor Herausnahme der Leber $400 \mathrm{mg}$ Galaktosamin $\cdot \mathrm{HCl}$ (Merck) pro kg Körpergewicht intraperitoneal (4).

Die Entnahme der Leber und die anschließende Perfusion erfolgte nach dem von Mrrler (2) und SCHIMASSEx (3) angegebenen Verfahren. Als Perfusionsmedium benutzten wir $100 \mathrm{ml}$ KREBsHensenett-Puffer, in den $5 \mathrm{~g}$ Rinderserumalbumin sowie $26 \mathrm{mg}$ Glucose und $50 \mu 15$ proz. Bromthalein gelöst war. Als Sauerstoffträger wurden in dieser Lösung $3 \mathrm{ml}$ des Fluorkohlenwasserstoffs
FC 751) durch Ultraschall einemulgiert, so daß eine stabile Suspension mit Partikeln unter $5 \mu \mathrm{m}$ Größe entsteht (5). Das Perfusat wurde ständig mit $\mathrm{O}_{2} / \mathrm{CO}_{2}=95: 5(\mathrm{v} / \mathrm{v})$ gesättigt. Während der Perfusion wurde zum Perfusat durch eine kontinuierliche Infusion Ammoniumchlorid zugegeben in Mengen zwischen 30 und $75 \mathrm{mg} / \mathrm{h} \cdot \mathrm{kg}$ Ratte. Bei einigen Versuchsgruppen wurde dem Perfusat noch kontinuierlich $100 \mathrm{mg} / \mathrm{h}$ Glucose zugefügt.

Ammoniak wurde enzymatisch nach dex Methode von ScHMidr und SCHWARz (6) bestimmt;

Harnstoff mit Diacetylmonoxim im Autoanalyser (7),

Glucose mit der Glucoseoxidase/Perid-Methode der Firma Boehringer (8), Kalium flammenphotometrisch.

Die Enzymaktivitäten bestimmen wir mit den Reagenzien der Firma Boehringer Alanintransaminase nach (9), Aspartattransaminase nach (10), Glutamatdehydrogenase nach (11), Lactatdehydrogenase nach (8). Bromthalein im Perfusat und in der Galle photometrisch; Bromthalein-Clearance aus der Abnahme der Bromthaleinkonzentration im Perfusat berechnet (10).

Die mathematische Behandlung der Ergebnisse erfolgt nach den statistischen Verfahren der Varianzanalyse (partiell hierarchisches Modell)2).

\section{Ergebnisse}

Vergleicht man die von uns gemessenen Parameter bei den normalen und geschädigten Lebern, dann ergibt sich, daß praktisch alle Größen durch die Galaktosamininjektion verändert werden. Obwohl wir jeweils die gleiche Dosis pro kg Körpergewicht an Galaktosamin verabreicht haben, konnten wir bei der Perfusion zwei Gruppen unterscheiden, eine Gruppe mit starker Abnahme aller von uns gemessenen Leberleistungen ver-

1) 3M-Company, Düsseldorf.

2) Wir danken dem Institut für Medizinische Statistik und Dokumentation, Gießen, (Leiter: Prof. Dr. J. DuDEck) für dïe Hilfe und Durchführung der statistischen Berechnung. 

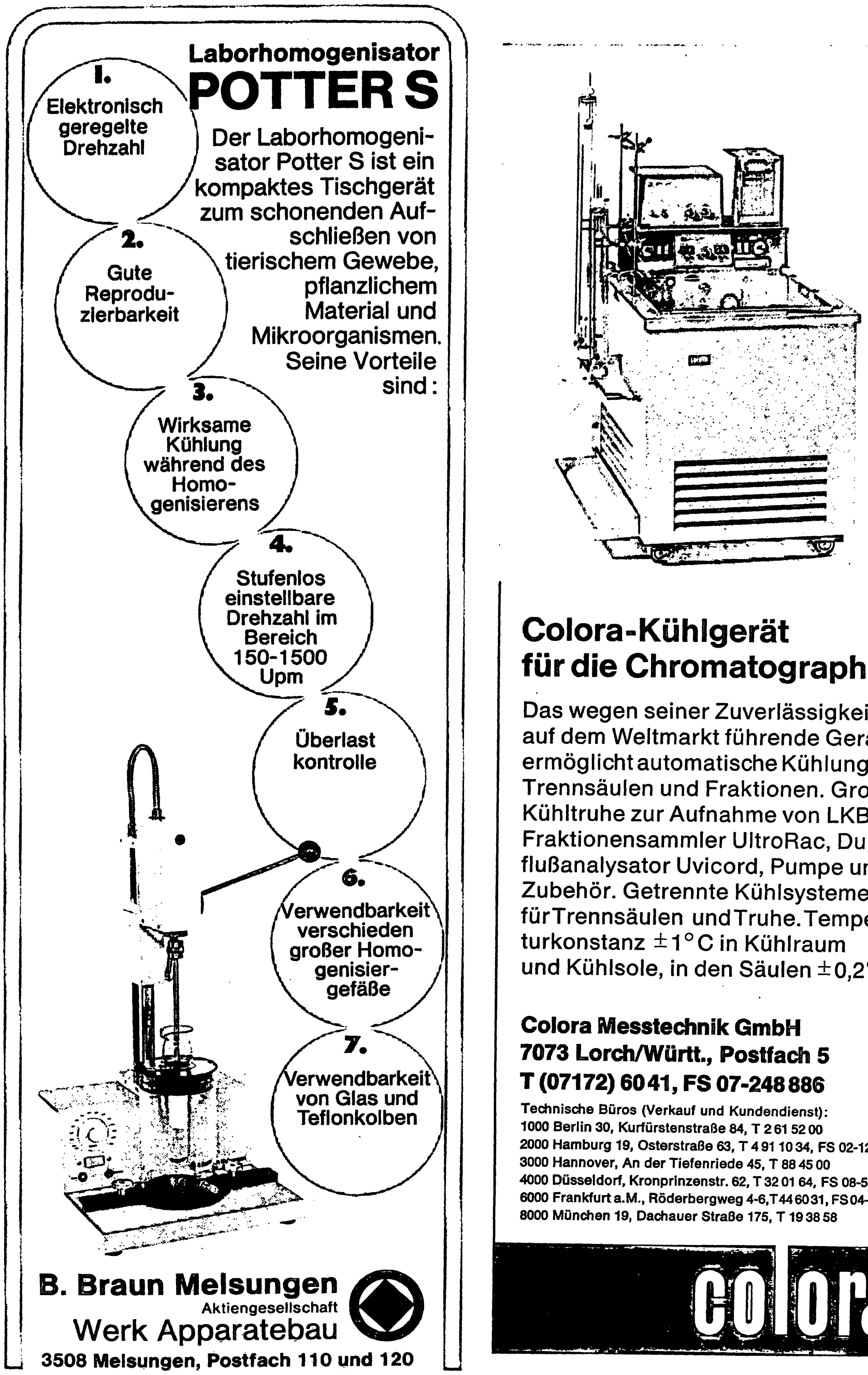

\section{Colora-Kühlgerät für die Chromatographie}

Das wegen seiner Zuverlässigkeit auf dem Weltmarkt führende Gerät ermöglicht automatische Kühlung der Trennsäulen und Fraktionen. Große Kühltruhe zur Aufnahme von LKBFraktionensammler UltroRac, Durchflußanalysator Uvicord, Pumpe und Zubehör. Getrennte Kühlsysteme fürTrennsäulen und Truhe. Temperaturkonstanz $\pm 1^{\circ} \mathrm{C}$ in Kühlraum und Kühlsole, in den Säulen $\pm 0,2^{\circ} \mathrm{C}$.

\section{Colora Messtechnik GmbH} 7073 Lorch/Württ., Postfach 5 T (07172) 6041, FS 07-248886

Technische Büros (Verkauf und Kundendienst): 1000 Berlin 30, Kurfürstenstraße 84, T 2615200 2000 Hamburg 19, Osterstraße 63, T 491 10 34, FS 02-12 947 3000 Hannover, An der Tiefenriede 45, T 884500 4000 Düsseldorf, Kronprinzenstr. 62, T 320164 , FS $08-587253$ 6000 Frankfurt a.M., Röderbergweg 4-6, T446031, FS04-11 216 8000 München 19, Dachauer StraBe 175, T 193858

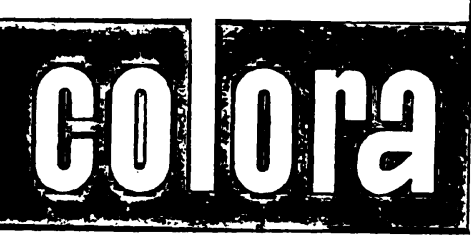




\section{HELWIG}

Zur Neuauflage 1972

Urteile der Fachpresse

\section{Moderne Arzneimittel}

\section{Eine Spezialitätenkunde nach Indikationsgebieten für Ärzte und Apotheker}

Von Dr. Burghard Helwig, Stuttgart, unter Mitarbeit von Dr. Helmut Helwig, Freiburg

4., völlig neubearbeitete und erweiterte Auflage 1972. Etwa 1650 Seiten. Gr.-8. Kunststoffeinband. Es gelten folgende Preise: Umtausch-Subskriptionspreis (gültig bis zum Erscheinen) bei Vorlage des Titelblattes der 3. Auflage DM 248.-; Subskriptionspreis (gültig bis zum Erscheinen) DM 272.-; Ladenpreis (gültig nach Erscheinen) DM 295.-

Dieses regelmäßig in hoher Auflage erscheinende Werk entspricht in der 4., völlig neubearbeiteten und erweiterten Auflage wiederum dem neuesten Stand der Arzneimittelkunde. Es kommt dem Wunsche von Ärzten und Pharmazeuten entgegen, eine noch größere aktuelle Transparenz in der Übersicht über die Vielzahl der verfügbaren Arzneimittel zu schaffen.

$\mathrm{Da}$ es für den Benutzer immer wichtiger wird, Näheres über die Indikations- oder Arzneimittelgruppe zu wissen, zu der das einzelne Präparat gehört, sind möglichst jẹder Gruppe vielfach umfassende Einleitungen und Erläuterungen z. T. mit Tabellen vorangestellt, die kritisch auf die Möglichkeiten und Grenzen der Therapie, auf die in Frage kommenden Wirkstoffe, deren Indikationen, mögliche erweiterte Angaben über Nebenwirkungen und Kontra-Indikationen eingehen. Gegebenenfalls wird hier auch auf Bekanntmachungen der Arzneimittelkommission der deutschen Ärzteschaft hingewiesen.

Die Einteilung nach Indikationsgruppen bzw. Arzneimittelgruppen wie Hormone, Vitamine, Sera, Röntgenkontrastmittel, Mittel zur Vergiftungsbekämpfung etc. wurde aưh in der Neuauflage beibehalten. Die bekannte Charakteristik der Arzneispezialitäten, durch die der HELWIG sich seither besonders auszeichnete, gibt detaillierte Schilderungen. Den Kontra-Indikationen wurde dabei noch mehr Bedeutung beigemessen als bisher.

„Das Buch bietet gerade für den Internisten und für die internistische Praxis mit ihrer besonderen Zuwendung zur Arzneitherapie eine brauchbare Arbeitsgrundlage und ist ein Nachschlagewerk, das eine vollständige Ubersicht über die am Markt befindlichen Präparate mit Urteilen über ihren therapeutischen Wert verbindet. “

(Der Internist) „Sowohl für den Apotheker in der Praxis als auch in der pharmazeutischen Forschung ist dieses Nachschlagewerk ein zuverlässiges und unentbehrliches Hilfsmittel.“ (Pharmaceutica Acta Helvetica)

WISSENSCHAFTLICHE VERLAGSGESELLSCHAFT MBH, D-70゙๊̣0 Stuttgart 1, Birkenwaldstraße 44, Pf. 40

\title{
BIOCHIMIE
}

\section{Edité par la Société de Chimie Biologique}

\author{
tel est le titre \\ sous lequel paraîtra à partir de 1971 \\ le „BULLETIN DE LA SOCIÉTÉ DE CHIMIE BIOLOGIQUE“
}

\section{SECRÉTARIAT}

de la Société de Chimie Biologique

J. P. EBEL, Secrétaire Général (Relations Extérieures)

R. PERLES, Secrétaire Général

\section{REDACTION}

F. GROS, Secrétaire scientifique

F. PERCHERON, Secrétaire à la Publication J. NUNEZ, Secrétaire à l'Information

Y. RAOUL, Secrétaire à l'Edition

SECRETARIAT et REDACTION: 4 Avenue de l'Observatoiré, PARIS $6^{\circ}$ 12 FASCICULES

ABONNEMENTS: FRANCE et ZONE FRANC: $150 \mathrm{ffrcs}$ - BELGIQUE: 1.687,- fres - AUTRES PAYS: 186,- ffrcs MASSON et Cie, Editeurs - 120 Boulevard St Germain · PARIS 6ème 
bunden mit einem starken Verlust an Leberzellenzymen, eine zweite Gruppe, bei der die Leberleistungen deutlich besser und die Enzymverluste deutlich geringer blieben. Worauf diese unterschiedliche Empfindlichkeit gegenüber Galaktosamin beruht, konnten wir nicht ermitteln. Eine Beziehung zum Körpergewicht der Ratten bestand nicht. Das Gewicht der Ratten mit schwerer Vergiftung betrug $221 \pm 20 \mathrm{~g}$, mit schwacher Vergiftung $219 \pm 23 \mathrm{~g}$.

Verglichen mit Normallebern sinkt entsprechend der Schwere der Vergiftung die Elimination von Ammoniak aus dem Perfusat (Abb. 1), während die Harnstoffsynthese sich nicht signifikant ändert (Abb. 2). Die normale Leber verwertet die angebotene Menge Ammoniak $(14,1 \mu \mathrm{mol} / \mathrm{h} \cdot \mathrm{g}$ Leber $)$ praktisch vollständig unter Einhaltung eines konstanten Ammoniumionenspiegels im Perfusat von rund $120 \mu \mathrm{mol} / \mathrm{l}$. Bei der ge-

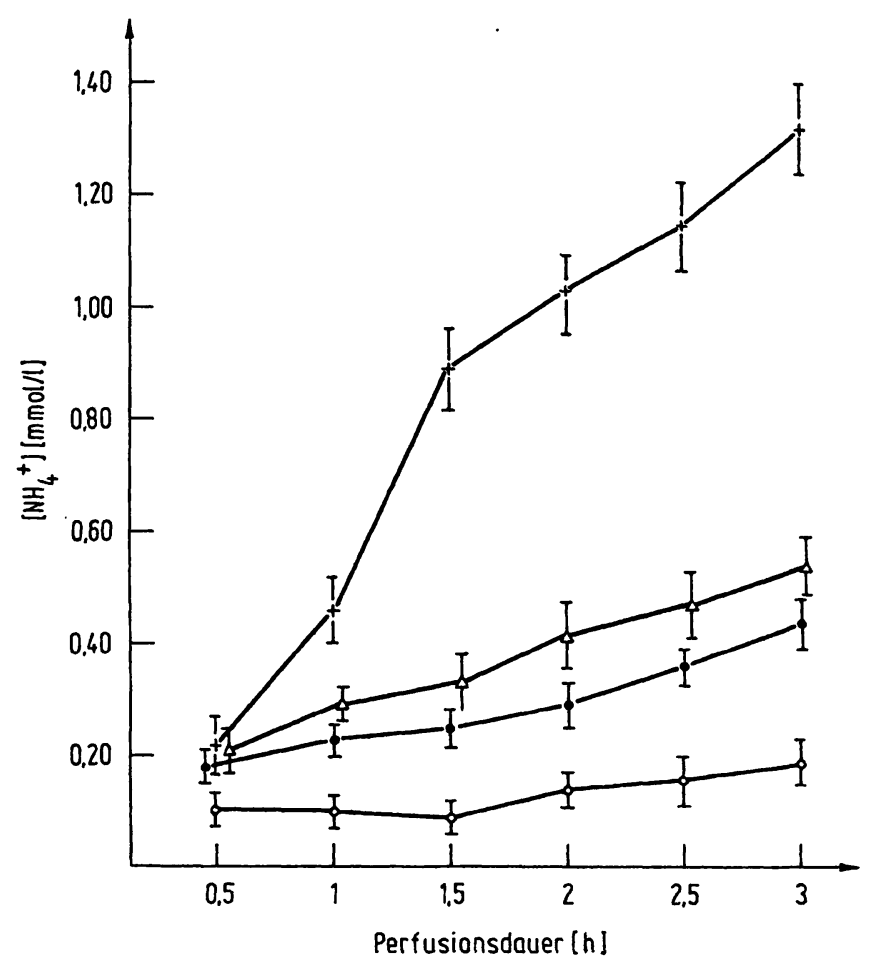

Abb. 1

Anstieg der Ammoniakkonzentration im Perfusionsmedium unter den verschiedenen Versuchsbedingungen

Kontrolle; $(\mathrm{n}=10) \pm 1 \mathrm{~s}$

schwach vergiftet; $(n=10) \pm 1$ s

+ stark vergiftet; $(\mathrm{n}=10) \pm 1 \mathrm{~s}$

$\triangle \quad$ stark vergiftet, Glucosezufuhr während der Perfusion $(n=10) \pm 1$ s

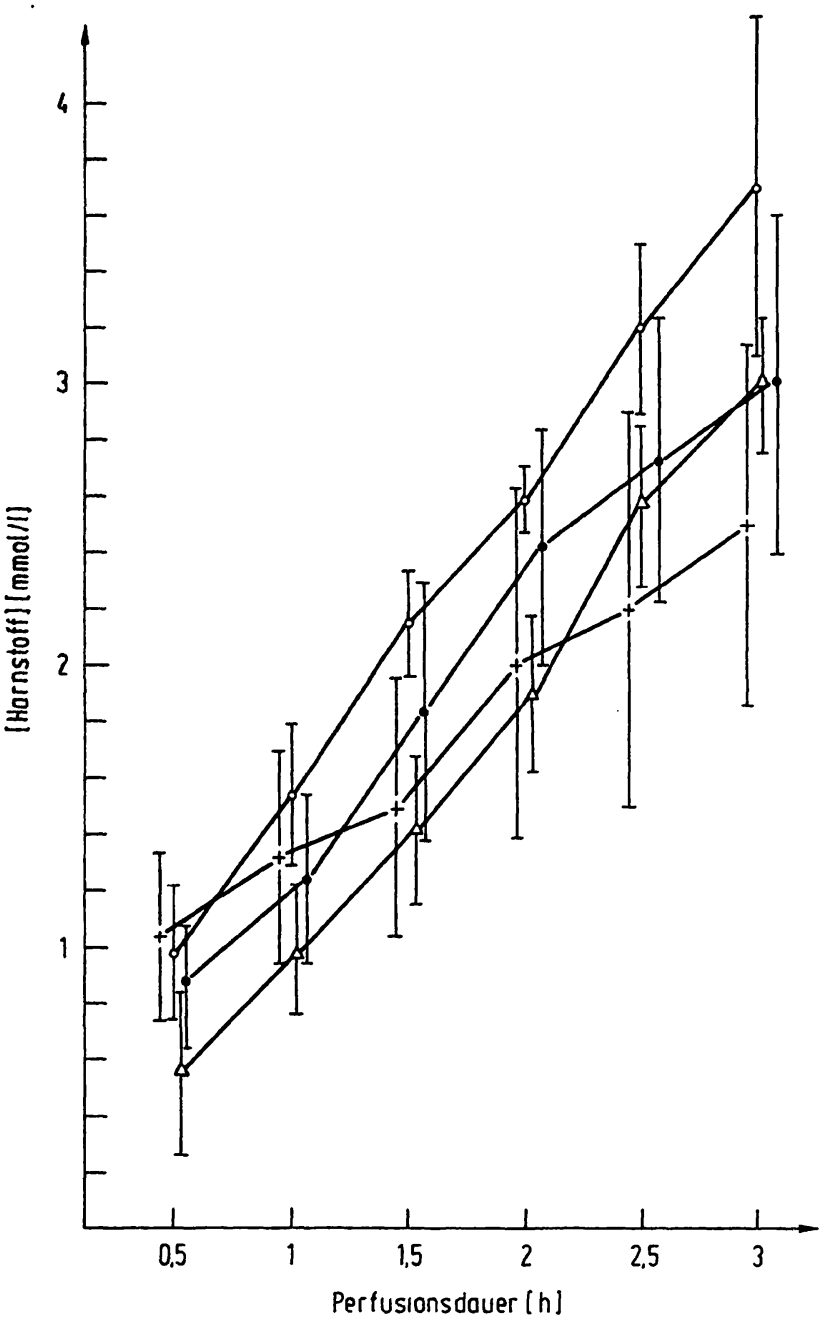

Abb. 2

Verhalten der Harnstoffhonzentration im Medium während der $3 \mathrm{~h}$ Perfusion unter den verschiedenen Versuchsbedingungen

O Kontrolle $(n=10) \pm 1 s$

schwach vergiftet $(n=10) \pm 1$ s

+ stark vergirtet $(n=10) \pm 1 \mathrm{~s}$

$\Delta$ stark vergiftet, Glucosezufuhr während der Perfusion $(n=10) \pm 1 \mathrm{~s}$

schädigten Leber kumulieren die Ammoniumionen im Perfusat kontinuierlich, so daß nach $3 \mathrm{~h}$ bei schwacher Schädigung ein Spiegel von $450 \mu \mathrm{mol} / \mathrm{l}$ erreicht wird, bei schwerer Schädigung $1400 \mu \mathrm{mol} / \mathrm{l}$. Während die Harnstoffsynthese bei der normalen Leber praktisch über die ganze Zeit von $3 \mathrm{~h}$ linear verläuft, sieht man bei den geschädigten Lebern nur $30 \mathrm{~min}$ lang eine nahezu normale Harnstoffsynthese, dann sinkt die Harnstoffsynthese entsprechend dem Schweregrad der

Tab. 1

Parallelbestimmungen von Ammoniakverwertung und Harnstoffsynthese. In allen Versuchsreihen wurden $14,1 \mu$ mol $\mathrm{NH}_{\mathrm{i}}^{+} / \mathrm{h} \cdot \mathrm{g}$ Leber angeboten

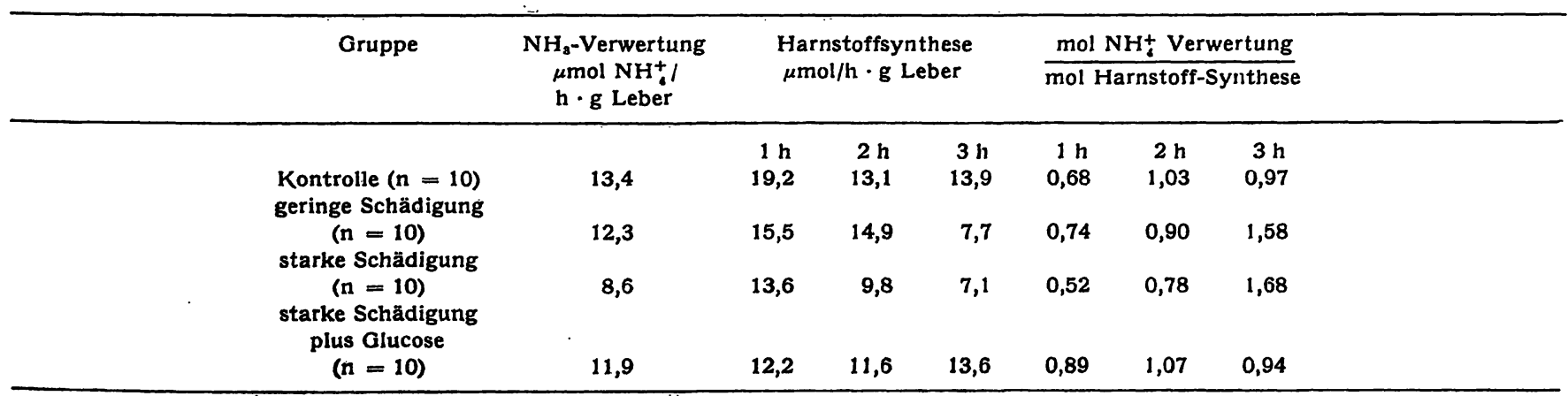


Schädigung ab. Bemerkenswert ist, daß der molare Quotient Ammoniak: Harnstoff, der bei der normalen Leber rund 1 beträgt (14), im Falle der Schädigung durch Galaktosamin im Verlauf der Perfusion bis über 1,6 ansteigt (Tab. 1).

Bei der Schädigung bleibt die Ammoniumionenclearance offenbar besser erhalten als die Harnstoffsynthese.

Aus unserer Versuchsordnung läßt sich nicht erkennen, $\mathrm{ob}$ in diesem Fall $2 \mathrm{~mol}$ Ammoniumionen in $1 \mathrm{~mol}$ Harnstoff umgebaut oder ob die zusätzlich eliminierten Ammoniumionen in Form eines Intermediärproduktes in der Leber gespeichert werden.

Solange die isoliert perfundierte Rattenleber nicht geschädigt ist, verläuft die Gallenproduktion stetig während $3 \mathrm{~h}$ (11). Nach Galaktosaminvergiftung ist die Gallenprodultion von Anfang an deutlicher vermindert (Abb. 3).

Die Bromthaleinclearance durch die Leber ist ein$\mathrm{mal}$ an eine ausreichende Kapillarisierung des Leberparenchyms gebunden, daneben an die Fähigkeit, BSP in die Parenchymzellen zu übernehmen, dort zu speichern, zu konjugieren und dann an die Galle abzugeben. Solange die Gallenproduktion noch intakt ist, kann daher geprüft werden, ob die Clearancefunktion für Bromthalein spezifisch eingeschränkt ist. Bei der Reduktion der Gallenproduktion dagegen kann eine verminderte BSP-Clearance allein aufgrund der Unfähigkeit der Leber, Galle zu produzieren, bedingt sein. Bei der Galaktosaminvergiftung geht die Bromthaleinclearance immer mit dem Verlust der Gallenproduktion parallel. Gleichzeitig mit dem Verlust der Gallenproduktion verlängern sich automatisch die BSP-Eliminationszeiten aus dem Perfusat von $T / 2=$ $67 \mathrm{~min}$ für die gesunde Leber auf $\mathrm{T} / 2=270 \mathrm{~min}$ bei schwacher und $\mathrm{T} / 2=750 \mathrm{~min}$ bei starker Vergiftung (Tab. 2).

Während die normale Leber Bromthalein in der Galle auf das 13,3fache konzentrieren kann, ist die höchste Konzentration, die wir nach schwacher Galaktosaminvergiftung gemessen haben, 9,3fach und nach starker Galaktosaminvergiftung 7,1fach.

Tabelle 2 zeigt, daß die Elimination von BSP bereits auf $25 \%$ der Norm absinkt, während die Konzentrationsleistung noch $72 \%$ der Norm beträgt. Auch bei starker Vergiftung ist die Konzentrationsleistung noch

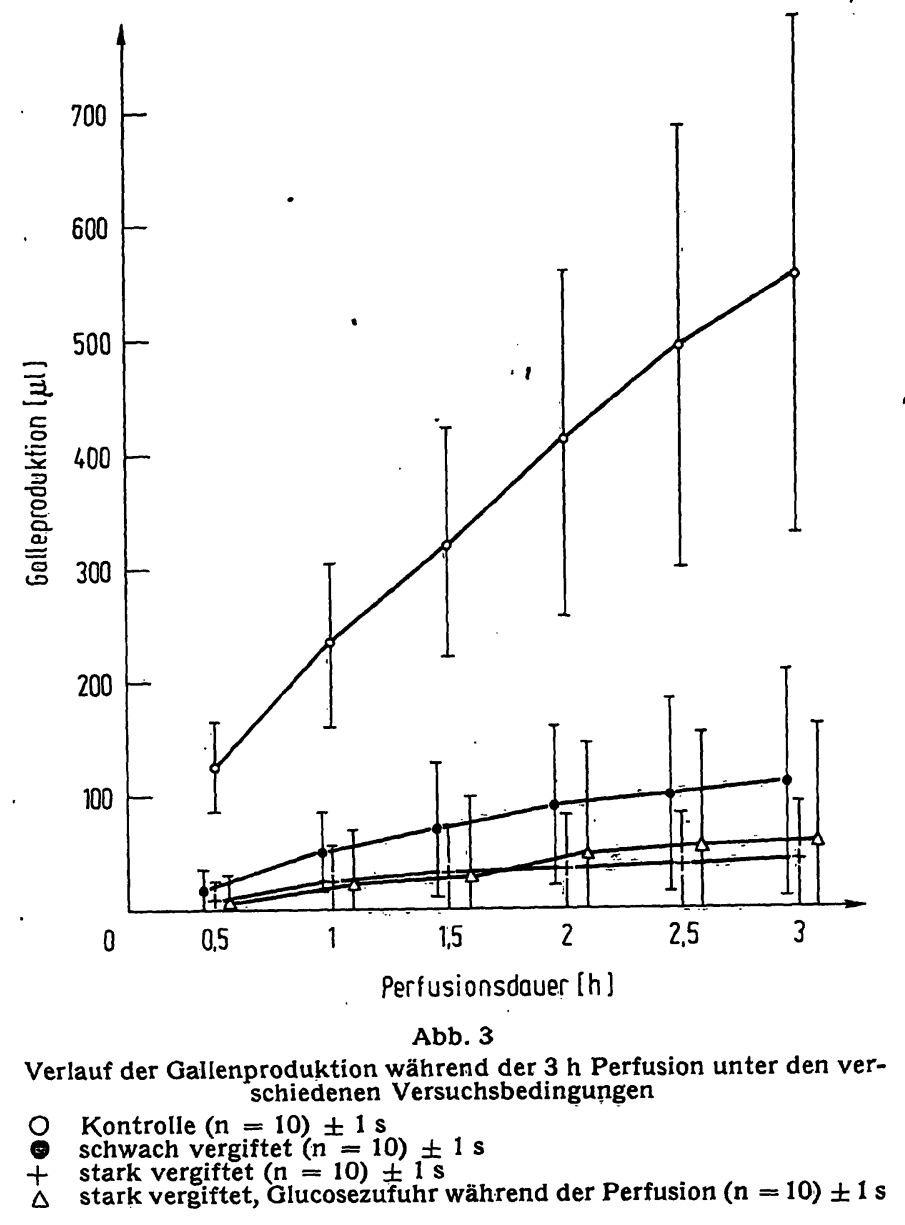

$54 \%$ der Norm, während der Eliminiationsfähigkeit auf $8,9 \%$ abgefallen ist.

Im Verlauf der Perfusion einer isolierten Rattenleber kommt es zu einer zunehmenden Zellschädigung, die sich darin äußert, daß zunehmend Kaliumionen und cytoplasmatische und schließlich mitochondriale Enzyme aus den Zellen an das Perfusat abgegeben werden. Aus der Geschwindigkeit des Anstieges dieser Enzyme und aus dem schließlich erreichten Enzymspiegel im Perfusat kann auf den Grad der Zellschädigung geschlossen werden. In unseren Versuchen mit gesunden Lebern beobachten wir-einen linearen Anstieg der Kaliumionenkonzentration im Perfusat von 4,48 $\mathrm{mmol} / \mathrm{l}$ auf 4,6 mmol/l. Dieser Anstieg bedeutet eine Abgabe von $1,5 \mu \mathrm{mol} \mathrm{Kalium} / \mathrm{g}$ Rattenleber (Tab. 3).

Auch der Austritt der cytoplasmatischen Enzyme Lactatdehydrogenase und Aspartattransaminase erfolgt

Tab. 2

Aus je 10 Versuchen errechnete Mittelwerte für die Gallenproduktion in $3 \mathrm{~h}$, maximale Konzentrationsfähigkeit für BSP (berechnet aus BSP-Konzentration in der Galle durch BSP-Konzentration im Perfusat) und Eliminationsgeschwindigkeit für BSP, errechnet aus der Abnahme der BSP-Konzentration im Perfusat

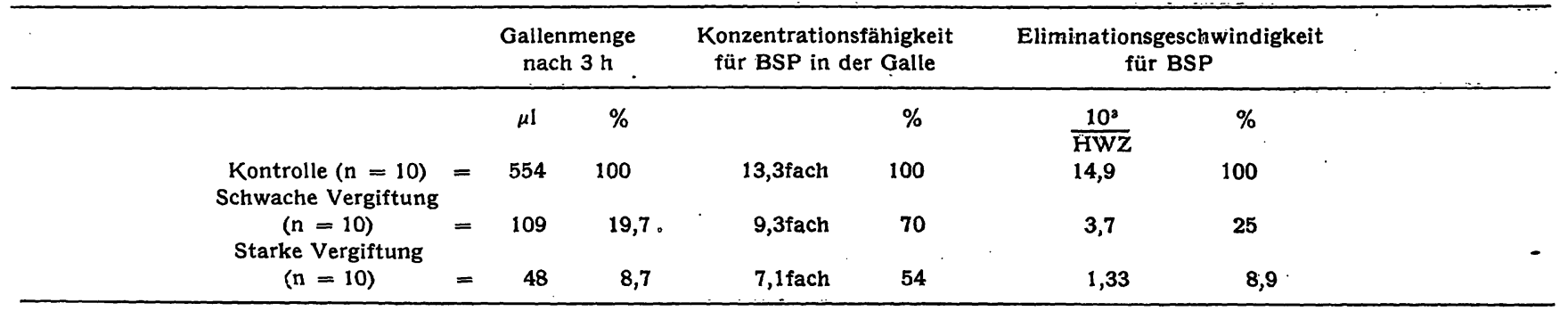


Tab. 3

Leberleistungen bei Galaktosaminvergiftung

\begin{tabular}{|c|c|c|c|c|c|}
\hline & Kontrolle & $\begin{array}{l}\text { schwach } \\
\text { vergiftet }\end{array}$ & $\begin{array}{l}\text { stark } \\
\text { vergiftet }\end{array}$ & Signifikanz & $\begin{array}{l}\text { stark vergiftet } \\
+ \text { Glucose }\end{array}$ \\
\hline Gallenproduktion $[\mu 1 / 3 \mathrm{~h}]$ & 554 & 109 & 48 & & 58 \\
\hline BSP-Clearance HWZ [min] & 67 & 270 & 750 & & 210 \\
\hline BSP-Konzentration in der Galle & 13,3 & 9,3 & 7,1 & & 12,7 \\
\hline Kalium [ $\mu \mathrm{mol} / \mathrm{g}$ Leber an Perfusat abgegeben] & 1,5 & 0,92 & 7,5 & $p<0,01$ & $-1,5$ \\
\hline $\begin{array}{c}{[\mathrm{mU} / \mathrm{h} \cdot \mathrm{g} \text { Leber an Perfusat abgegeben] }} \\
\text { Alanintransaminase }\end{array}$ & 164,5 & 599,7 & - 1469,7 & $\mathrm{p}<0,01$ & 797,8 \\
\hline $\begin{array}{c}\text { [mU/h } \cdot \mathrm{g} \text { Leber an Perfusat abgegeben }] \\
\text { Aspartattransaminase }\end{array}$ & 15,6 & 41,4 & 169,9 & $p<0,01$ & 62,8 \\
\hline $\begin{array}{c}\text { [mU/h } \cdot g \text { Leber an Perfusat abgegeben] } \\
\text { Glutamatdehydrogenase }\end{array}$ & 91,6 & 126,7 & 549,5 & $p<0,01$ & 169,6 \\
\hline [mU/h $\cdot g$ Leber an Perfusat abgegeben] & 2,0 & 3,8 & 11,2 & $\mathrm{p}<0,01$ & 3,9 \\
\hline Glucose [mmol im Perfusat nach $3 \mathrm{~h}$ ] & 5,6 & 0,78 & 0,34 & $\mathrm{p}<0,01$ & 9,7 \\
\hline Ammoniak $[\mu \mathrm{mol} / \mathrm{l}$ im Perfusat nach $3 \mathrm{~h}]$ & 18,7 & 43,8 & 131,8 & $\mathrm{p}<0,01$ & 54,1 \\
\hline Harnstoff $[\mu \mathrm{mol} / \mathrm{l}$ im Perfusat nach $3 \mathrm{~h}]$ & 370 & 305 & 251 & n.s. & 300 \\
\hline
\end{tabular}

Angegeben sind die Mittelwerte aus je 10 Versuchen n. s. = nicht signifikant

linear über $3 \mathrm{~h}$. Aus dem Anstieg der Enzymkonzentration im Perfusat folgt, daß pro Stunde und pro $g$ Leber $165 \mathrm{mU}$ Lactatdehydrogenase und $15,6 \mathrm{mU}$ Alanintransaminase freigesetzt werden. Aspartattransaminase als partiell cytoplasmatisch und partiell mitochondriales Enzym verhält sich ähnlich wie die rein cytoplasmatischen Enzyme. Hier werden $91,6 \mathrm{mU} / \mathrm{h} \cdot \mathrm{g}$ Leber abgegeben. Vom rein mitochondrialen Enzym Glutamatdehydrogenase treten während der Perfusion der gesunden Leber $2 \mathrm{mU} / \mathrm{h} \cdot \mathrm{g}$ Leber in das Perfusat über.

Bei der Perfusion der Lebern von Ratten, die Galaktosamin erhalten hatten, erkennt man sofort die wesentlich stärkere Zellschädigung:

Der Kaliumspiegel erreicht im Mittel $5,5 \mathrm{mmol} / \mathrm{l}$, an Enzymen werden pro $h$ und $g$ Leber abgegeben: Lactatdehydrogenase $1470 \mathrm{mU}$, Alanintransaminase $170 \mathrm{mU}$, Aspartattransamiase $550 \mathrm{mU}$, Glutamatdehydrogenase $11,2 \mathrm{mU}$.

Über die Regulation des Glucosestoffwechsels in der isoliert perfundierten Rattenleber ist bekannt (15), daß die isolierte Leber in der Lage ist, einen konstanten Glucosespiegel im Perfusat einzustellen, gleichgültig ob man mit dem Perfusat Glucose anbietet oder nicht. Auch in unseren Versuchen fanden wir, daß die normale Leber beim Angebot von $260 \mathrm{mg} / 1$ Glucose im Perfusat innerhalb kurzer Zeit einen Spiegel von rund $1000 \mathrm{mg} / \mathrm{l}$ einstellt und diesen Spiegel bis zum Ende des Versuches aufrecht erhält. Die galaktosamingeschädigte Leber hat diese Fähigkeit verloren.

Bei den schwachen Vergiftungen sinkt der Glucosespiegel im Perfusat von einer Ausgangskonzentration von $250 \mathrm{mg} / \mathrm{l}$ auf $145 \mathrm{mg} / \mathrm{l}$. Die stark geschädigten Lebern können dagegen auch diesen Spiegel nicht aufrecht erhalten, vielmehr sinkt die Glucosekonzentration im Perfusat im Durchschnitt bis auf $61 \mathrm{mg} / 1$, in einer Reihe von Versuchen bis auf 0 ab. Galaktosamin fixiert UTP, so daß keine UDPG für die Neusynthese von Glykogen zur Verfügung steht (13). Die Galak- tosaminleber verarmt dadurch an Glykogen. Bietet man der Galaktosaminleber mit dem Perfusat $100 \mathrm{mg}$ Glucose pro $\mathrm{h}$ an, nimmt sie davon rund $50 \mathrm{mg}$ pro $\mathrm{h}$ auf. Damit parallel bessert sich die Leistung, erkennbar insbesondere an der vermehrten Synthese von Harnstoff. Auch die Zellschädigung ist schwächer, erkennbar an dem geringeren Enzymverlust der Leberzellen (Tab. 3).

\section{Diskussion}

Eine Galaktosaminleber läßt sich ebenso wie eine Normalleber isoliert perfundieren. Gegenüber der Normalleber ist dabei die Syntheseleistung und die Gallenproduktion deutlich vermindert. Die Regulation des Glucosespiegels im Perfusat versagt. Als Zeichen der Parenchymzellschädigung ist der Enzymaustritt auf das Vielfache der Norm angestiegen (Tab. 3).

Bei der galaktosamingeschädigten Leber sinkt die Harnstoffsynthese stärker als die Ammoniakelimination, so daß schließlich ein molares Verhältnis Ammoniak: Harnstoff von nahezu 2 resultiert. Während normalerweise aus dem Gesamtbestand der Leber während der 3stündigen Perfusion nur 0,8\% Kaliumionen und - berechnet aus den Werten von Schmidt (12) für den Enzymgehalt in der Leber - von Lactatdehydrogenase $0,14 \%$, von Alanintransaminase $0,31 \%$, von Aspartattransaminase 0,25\% und vom mitochondrialen Enzym Glutamatdehydrogenase nur 0,013\% austreten, verliert die Leber nach Galaktosaminschädigung 4,0\% ihres Kaliums, 1,25\% ihres Bestandes an Lactatdehydrogenase, $3,38 \%$ an Alanintransaminase, $1,5 \%$ an Aspartattransaminase und $0,073 \%$ an Glutamatdehydrogenase.

Die Beobachtung, daß durch zugeführte exogene Glucose die Funktion der Galaktosaminleber deutlicher verbessert wurde, läßt vermuten, daß der Funktionsverlust sekundär infolge des Glykogenmangels auftritt, während exogen angebotene Glucose verwertet wird und die für die Leistungen erfordcrliche Energie liefert. 


\section{Literatur}

1. Keppler, D., Lesch, R., Reutter, W. \& Decker, K. (1968), Experimental and Molecular Pathology 9, 279-290. - 2. MrLLER, L. L. \& Watson, M. L. (1951), J. Exp. Med. 94, 431-453. - 3. SCHIMASSEK, H. (1963), Biochem. Z. 336, 460-467. - 4. KePPLER, D., LesCh, R., Reutter, W. \& DeCKer, K. (1970), HoppeSeyler's Z. Physiol. Chem. 351, 102-104. - 5. GeYeR, R. P. (1970), Medizin und Ernährung 11, 256-261. - 6. Schmrdt, F. H., Schwarz, M. (1966), Klin. Wochenschr. 44, 591-592. - 7. Marsh, W. H., Fingerhut, B. \& Miller, H. (1965), Clin. Chem. 11, 624 627. - 8. Bergmeyer, H. U. \& Bernt, E. (1970), in Methoden der enzymatischen Analyse (Hrsg. BeR GMEYER, H. U.) 2. Aufl. S. 1179-1180, Verlag Chemie Weinheim/Bergstr.. - 9. Bergmeyer, H. U. \& BerNt, E. (1970), in 1. c. (8), S. 685 . 10. Richterich, R. (1968), Klinische Chemie, (Hrsg. R. Rrch-
TORICH), 2. Aufl., S. 317-319, Akademische Verlagsgesellschaft, Frankfurt/Main. - 11. Brauer, R. W. \& Pesottr, R. L. (1951), Proc. Soc. Exp. Biol. Med. 78, 174-181. - 12. Scrmidt, E., Schmidt, F. W., Herforth, C., Detrmar, K. H. \& Fabel, H. (1966), Enzymol. Biol. Clin. 7, 167-184. - 13. DeCker, K., KepPLER, D., RüDIGER, J. \& DOMSCHKE, E. (1971), Hoppe-Seyler's Z. Physiol. Chem. 352, 412-418. - 14. RókA, L. \& MIERTSCH, H. J. (1972), diese Z. 10, 416-419. - 15. SchrmasseK, H. (1963), Biochem. Z. 336, 460-467. - 16. BERGMEYER, H. U. \& BERNT, E. (1970), GPT; UV-Test mit Lactat-Dehydrogenase als Indikator Enzym in 1. c. (8), S. 717. - 17. SchMIDT, E. (1970), GLDH; UV-Test in 1. c. (8), S. 607. - 18. BERGMEYER, H. U. \& BERNT, E. (1970), LDH; UV-Test mit Pyruvat und NADH in 1. c. (8), S. 533.
Prof. Dr. L. Róka 6300 Gießen

Klinikstr. 32b 


\section{Was werfen}

\section{Sie lieber weg:}

„Piepen" oder Pipetten?

Schon heute kostet das Reinigen von Mikropipetten mehr Zeit und Geld, als der Gebrauch von Einmal-Kapillarpipetten.

Was also liegt näher, als EinmalKapillarpipetten-Blaubrand zu nehmen?

So selbstverständlich, wie Streichhölzer und Trinkhalme. Mehr darüber auf́ der nächsten Seite.
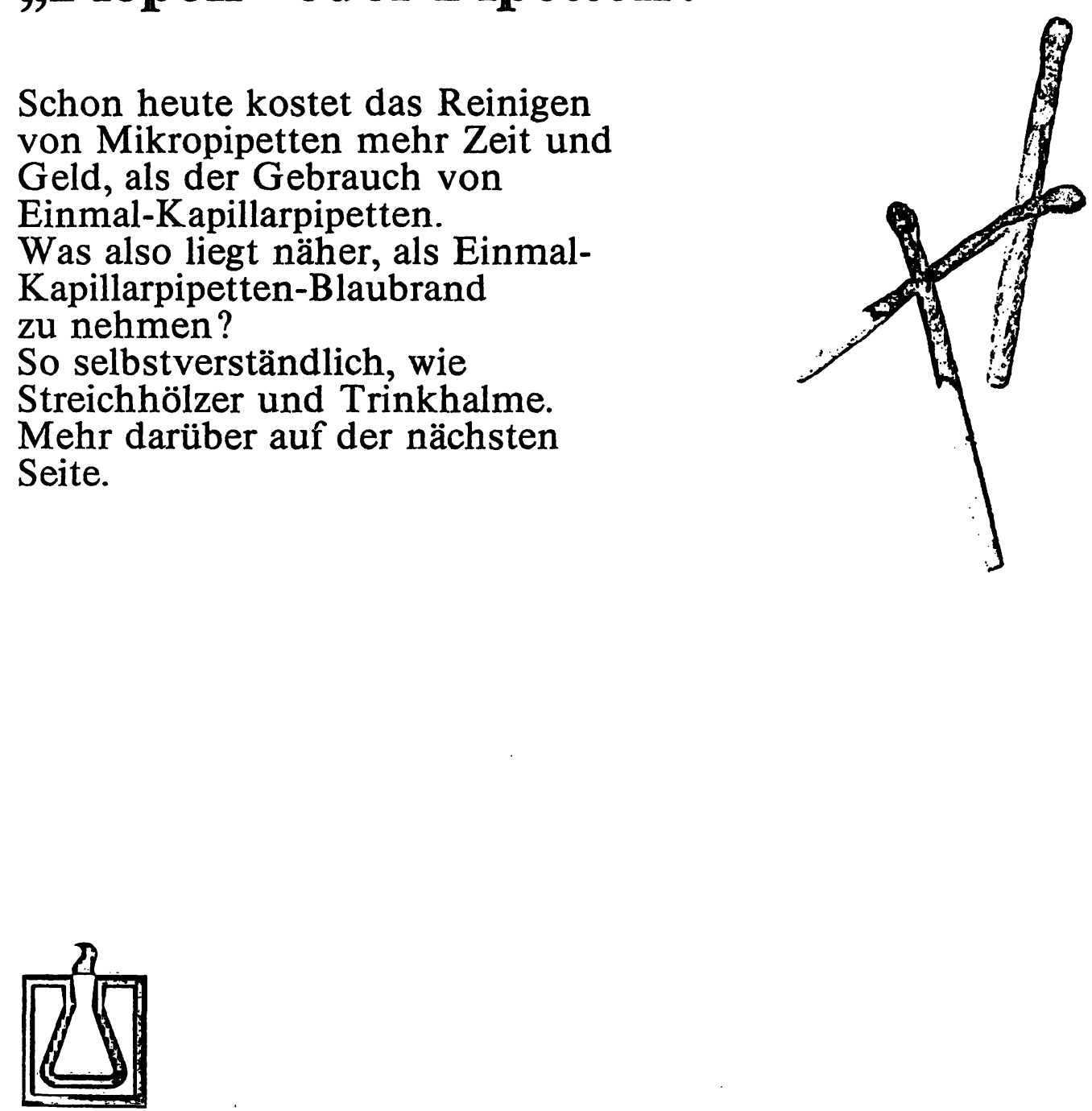


\section{|| || || || |||| Color-Code}

BLAUBRAND-Mikropipetten mit Ringmarke für den Einmalgebrauch tragen den Color-Code nach DIN 12621 für Vollpipetten, daher sind Verwechselungen ähnlich großer Pipetten ausgeschlossen.

\section{Genauigkeit}

Aufgrund der Verwendung von Präzisionskapillaren ist die Zuverlässigkeit der BLAUBRAND-Mikropipetten für den Einmalgebrauch außerordentlich gut.
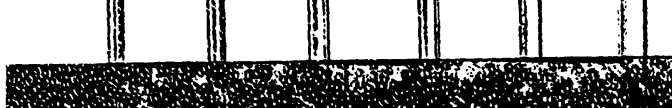

Die Fehler liegen in aller Regel erheblich unterhalb der Eichfehlergrenze.

Sie liegen sogar in aller Regel wesentlich unterhalb $1 \%$.

Die BLAUBRAND-Mikropipetten für den Einmalgebrauch sind gemäß $\$ 6.1 .1$. der Eichpflichtausnahmeverordnung von der Eichpflicht Stück für Stück ausgenommen da sie den einschlägigen Vorschriften der Eichordnung sinngemäß .entsprechen.

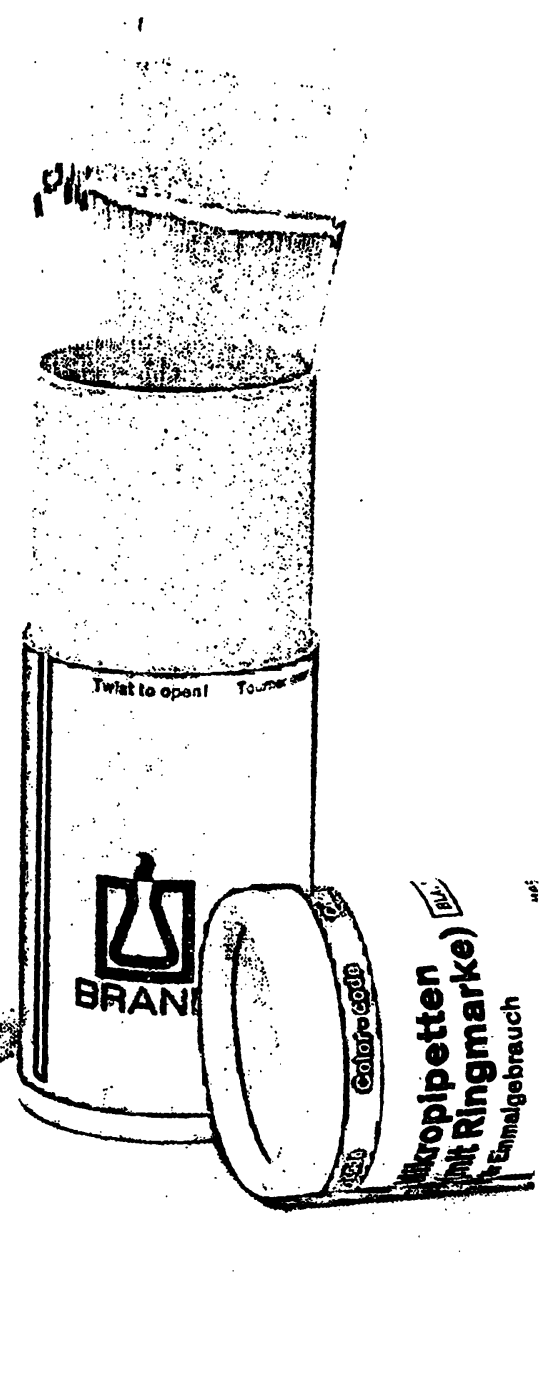

\section{Wirtschaftlichkeit}

Kostenanalysen in verschieden weit automatisierten Laboratorien haben gezeigt daß die Reinigung von Pipetten - und zwar speziell im Mikroliter- Bereich ein sehr kostenintensiver Arbeitsvorgang ist. Je nach dem Grade der Automation kostet es zwischen 27 und 35 Pfennig, Kapillarpipetten zu reinigen, zu trocknen und gegeberienfalls zu sterilisieren. Demgegenüber erfordert die Benutzung von Einmal-Pipetten im ungünstigsten Falle nur $63 \%$ der Kosten für die Reinigung und Wiederverwendung konventioneller Pipetten, im günstigsten Falle sogar nur $31 \%$ dieser Kosten.

\begin{tabular}{|c|c|c|c|}
\hline Art.-No. & Inhalt & Tol. & Color-Code \\
\hline $\begin{array}{l}708705 \\
708709\end{array}$ & $\begin{array}{r}5 \mu l \\
10 \mu l\end{array}$ & $\begin{array}{l} \pm 2 \% \\
\pm 1 \%\end{array}$ & $\begin{array}{l}\text { weiß } \\
\text { orange }\end{array}$ \\
\hline
\end{tabular}

\begin{tabular}{clll} 
Art.-No. & Inhalt & Tol. & Color-Code \\
\hline 708718 & $20 \mu 1< \pm 1 \%$ & schwarz \\
708722 & $25 \mu \mid< \pm 1 \%$ & $2 \times$ weiß \\
708733 & $50 \mu 1< \pm 1 \%$ & grün \\
\hline
\end{tabular}

$708733 \quad 50 \mu 1< \pm 1 \%$ grün

bis 11 Pckg.

ab 36 Pckg. ( 9 Überkart.) DM 32,ab 120 Pckg. ( 30 Überkart.) DM 30,ab 200 Pckg. (50 Überkart.) DM 28,-

Art.-No. Inhalt Tol. Color-Code

$708744100 \mu 1< \pm 1 \%$ blau

bis 11 Pckg.

DM 36,ab 12 Pckg. ( 3 Überkart.) DM 35,ab 36 Pckg. ( 9 Überkart.) DM 33,ab 120 Pckg. (30 Uberkart.) DM 31,ab 200 Pckg. (50 Uberkart.) DM 29,-

Die Preise für die Einmalpipetten sind Nettopreise + MWST und verstehen sich pro Packung von 250 Stück. Die
Staffelpreise beziehen sich auf Abnahm pro Größe. Packungen mit Einmalpipet ten verschiedener Größe können nich addiert werden, um zu einem günstige ren Staffelpreis zu gelangen.

\section{COUPON}

Ausführliche Informationen erhalten Sie, wenn Sie diesen Coupon ausschneiden und einsenden.

Wünschen Sie Muster zu erhalten, so kreuzen Sie bitte die gewünschte Größe an:

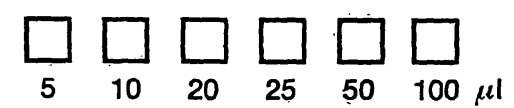

Sie erhalten 1 Musterpackung kostenlos. Weitere Musterpackungen werden zum Unkostenbeitrag von DM 1,- p. Packung + MWSt abgegeben: 